\title{
Intersection Properties of Simple Random Walks: A Renormalization Group Approach
}

\author{
G. Felder and J. Fröhlich \\ Theoretical Physics, ETH-Hönggerberg, CH-8093 Zürich, Switzerland
}

\begin{abstract}
We study estimates for the intersection probability, $g(m)$, of two simple random walks on lattices of dimension $d=4,4-\varepsilon$ as a problem in Euclidean field theory. We rigorously establish a renormalization group flow equation for $g(m)$ and bounds on the $\beta$-function which show that, in $d=4, g(m)$ tends to zero logarithmically as the killing rate (mass) $m$ tends to zero, and that the fixed point, $g^{*}$, in $d=4-\varepsilon$ is bounded by const $^{\prime} \varepsilon \leqq g^{*} \leqq \operatorname{const} \varepsilon$. Our methods also yield estimates on the intersection probability of three random walks in $d=3,3-\varepsilon$. For $\varepsilon=0$, these results were first obtained by Lawler [1].
\end{abstract}

\section{Introduction}

Two Brownian paths in $\mathbb{R}^{d}$ starting at different points intersect with positive probability in less than four dimensions, but do never intersect in four or more dimensions [2,3].

The continuum limit of $g_{0}|\phi|_{d}^{4}$ theory, $\phi=\phi$, or $\phi=\left(\phi^{1}, \phi^{2}\right), g_{0}>0$, is an interacting theory in less than four dimensions, in the superrenormalizable regime [4], but is a (generalized) free field in more than four dimensions [5]. Results in four dimensions remain incomplete, but there are strong reasons to expect that the continuum limit is trivial in that case, too.

Symanzik recognized the connection between these two facts in his work [6] on a representation of $g_{0}|\phi|^{4}$-theory as a gas of Brownian paths with local, repulsive interaction. Further work on that connection led to a novel, rather intuitive approach to scalar quantum field theory to which several people contributed valuable results, in the past few years. (See e.g. [7] and references therein for reviews of recent results.)

On a more abstract, more heuristic level, much insight into the theory of critical points in lattice field theories and the related problem of constructing continuum limits in dimensions close to four has been accomplished by using renormalization group methods; see e.g. [8] and references given there. In particular, for $g_{0}|\phi|^{4}$-theories, perturbative renormalization group equations predict that, in four dimensions, the renormalized coupling constant $g=g(m)$ tends to zero like 
$|\ln m|^{-1}$, as the mass $m$ (or, equivalently, the lattice spacing) tends to 0 , while in $d=4-\varepsilon$ dimensions $g(m)$ approaches a fixed point $g^{*}=0(\varepsilon)$, as $m \searrow 0$. Unfortunately, it has not been possible, so far, to find rigorous renormalization group flow equations which would yield mathematical proofs of these facts. It may be a little interesting, therefore, that, in estimating intersection probabilities of simple random walks, one can successfully use rigorous renormalization group flow equations. This is demonstrated in the following. Hence, while it has been shown already that random-walk methods are powerful tools to study field theory, it is now known that field-theoretic methods are useful in the study of random walk problems.

It is worthwhile and amusing to first pose the problem of estimating intersection probabilities of random walks explicitly as a problem in Euclidean field theory. This will show how and why heuristic renormalization group methods can be applied to random-walk problems.

Consider a lattice field theory of two interacting $N$-component fields, $\phi=\left(\phi^{1}, \ldots, \phi^{N}\right)$ and $\psi=\left(\psi^{1}, \ldots, \psi^{N}\right)$, with action

$$
\begin{aligned}
\mathscr{A}(\boldsymbol{\phi}, \psi)= & \sum_{x \in \mathbb{Z}^{d}}\left\{\frac{1}{2}\left|(\nabla \phi)_{x}\right|^{2}+\frac{1}{2}\left|(\nabla \psi)_{x}\right|^{2}+\frac{m^{2}}{2}\left(\left|\phi_{x}\right|^{2}+\left|\psi_{x}\right|^{2}\right)\right. \\
& \left.+\frac{g_{0}}{4}\left|\phi_{x}\right|^{2}\left|\psi_{x}\right|^{2}+\frac{g_{0}^{\prime}}{4}\left(\left|\phi_{x}\right|^{4}+\left|\psi_{x}\right|^{4}\right)\right\} .
\end{aligned}
$$

Such models were studied from the point of view of $\varepsilon$-expansions by Brézin et al. [9]. Let $\langle(\cdot)\rangle$ denote the Euclidean vacuum expectation for the theory with action $\mathscr{A}(\phi, \psi)$ given by (1.1). We define a susceptibility $\chi$ as

$$
\chi=\sum_{x \in \mathbb{Z}^{d}}\left\langle\phi_{0}^{1} \phi_{x}^{1}\right\rangle=\sum_{x \in \mathbb{Z}^{d}}\left\langle\psi_{0}^{1} \psi_{x}^{1}\right\rangle,
$$

the physical mass, $m_{\text {phys }}$, as the exponential decay rate of $\left\langle\phi_{0}^{1} \phi_{x}^{1}\right\rangle$ in $|x|$, and physical coupling constants $g$ and $g^{\prime}$ by

$$
g=-\bar{u}_{4} \chi^{-2} m_{\text {phys }}^{d}, g^{\prime}=-\bar{u}_{4}^{\prime} \chi^{-2} m_{\text {phys }}^{d},
$$

where

$$
\begin{gathered}
\bar{u}_{4}=\sum_{x, y, z}\left\langle\phi_{0}^{1} \phi_{x}^{1} ; \psi_{y}^{1} \psi_{z}^{1}\right\rangle, \\
\bar{u}_{4}^{\prime}=\sum_{x, y, z}\left\langle\phi_{0}^{1} ; \phi_{x}^{1} ; \phi_{y}^{1} ; \phi_{z}^{1}\right\rangle,
\end{gathered}
$$

and $\left\langle A_{1} ; A_{2} ; \ldots\right\rangle$ denotes a connected expectation. In [9] renormalization group fixed points for $g$ and $g^{\prime}$ have been computed as functions of $N$, to first order in $\varepsilon=4-d$. The result is:

$$
g^{*}=\frac{(4-N) \varepsilon}{2 N(N+8)-16(N-1)}, \quad g^{*}=\frac{N \varepsilon}{2 N(N+8)-16(N-1)} .
$$

Thus $g^{\prime *}$ and hence $g^{\prime}$ tend to 0 , as $N \rightarrow 0$ [in the domain of attraction of the fixed point (4)]. In the limit $N=0$, the flow equation for $g$ as a function of $m_{\text {phys }}=m$ is 
given by

$$
-\frac{d g}{d|\ln m|}=\beta(g), \quad \text { where } \quad \beta(g)=-\varepsilon g+4 g^{2}+0\left(g^{3}\right)
$$

(Note that the roots of $\beta$ are $g=0, g=g^{*}$, to first order in $\varepsilon$.)

Surprisingly, the $N \rightarrow 0$ limit of the theories considered here is a theory of Brownian paths with a local, repulsive interaction between any two paths. For the lattice theory this is easy to understand: Following [10] one may use a random-walk representation of that theory to show that

$$
\begin{aligned}
& \lim _{N, g_{0}^{\prime} \rightarrow 0}\left\langle\phi_{x_{1}}^{\alpha_{1}} \ldots \phi_{x_{2 n}}^{\alpha_{2 n}} \psi_{y_{1}}^{\beta_{1}} \ldots \psi_{y_{2 k}}^{\beta_{2 k}}\right\rangle \\
& \quad=\sum_{p, q} \sum_{\substack{\omega_{1}, \ldots, \omega_{n} \\
\omega_{1}^{\prime}, \ldots, \omega_{k}^{\prime}}} \int \ldots \int \prod_{i=1}^{n} d v_{\omega_{i}}^{m}\left(t^{i}\right) \prod_{j=1}^{k} d v_{\omega_{j}^{\prime}}^{m}\left(s^{j}\right) \exp \left(-g_{0} \sum_{x} \sum_{i, j} t_{x}^{i} \cdot s_{x}^{j}\right),
\end{aligned}
$$

where the (nearest-neighbor) walks $\omega_{i}, i=1, \ldots, n$ have endpoints $x_{p(2 i-1)}, x_{p(2 i)}$ with $\alpha_{p(2 i-1)}=\alpha_{p(2 i)}$, and, similarly, the walks $\omega_{j}^{\prime}$ connect $y_{q(2 j-1)}$ to $y_{q(2 j)}$, where $\beta_{q(2-1)}=\beta_{q(2 j)} ; p$ and $q$ are paitings of $\{1, \ldots, 2 n\},\{1, \ldots, 2 k\}$, respectively. The lattice fields $t^{i}=\left\{t_{x}^{i}\right\}_{x \in \mathbb{Z}^{d}}$ and $s^{j}=\left\{s_{x}^{j}\right\}_{x \in \mathbb{Z}^{d}}$ are waiting (or local) times associated with $\phi$ and $\psi$, respectively. Their a priori-distributions are given by

$$
\begin{gathered}
d v_{\omega}^{m}(t)=\prod_{x \in \mathbb{Z}^{d}} d v_{n_{x}(\omega)}\left(t_{x}\right) \exp \left(-\left(2 d+m^{2}\right) t_{x}\right), \\
d v_{n}(t)= \begin{cases}\delta(t) d t, & n=0 \\
\frac{t^{n-1}}{(n-1) !} \theta(t) d t, & n \geqq 1,\end{cases}
\end{gathered}
$$

and $n_{x}(\omega)$ is the total number of visits of $\omega$ at the site $x$.

It should be noted that for the lattice $g_{0}|\phi|^{2}|\psi|^{2}$-theory in finite volume an analytic interpolation in $N$ follows immediately from Symanzik's representation $[6,10]$, and a rigorous proof of (1.6) is a straight-forward consequence of the formulas in [10]. This has first been noticed in [11]. Important special cases of (1.6) are:

$$
\begin{aligned}
G_{m}(x, y) & \equiv \lim _{N, g_{0}^{\prime} \rightarrow 0}\left\langle\phi_{x}^{1} \phi_{y}^{1}\right\rangle=\lim _{N, g_{0}^{\prime} \rightarrow 0}\left\langle\psi_{x}^{1} \psi_{y}^{1}\right\rangle \\
& =\sum_{\omega: x \rightarrow y} \int d v_{\omega}^{m}(t)=\sum_{\omega: x \rightarrow y} \xi^{|\omega|+1},
\end{aligned}
$$

where $\xi \equiv\left(2 d+m^{2}\right)^{-1},|\omega|=\#$ jumps of $\omega$. This follows directly from (1.6) and (1.7). The right-hand side of (1.8) is well-known to be the Green's function of $-\Delta+m^{2}$, where $\Delta$ is the finite difference Laplacian. From (1.8) and the simple identity

$$
\left[e^{T\left(\Delta-m^{2}\right)}\right]_{x y}=\sum_{\omega: x \rightarrow y} \int d v_{\omega}^{m}(t) \delta\left(T-\sum_{x} t_{x}\right)
$$


we infer that $d v_{\omega}^{m}(t)$ is the waiting-time representation of the discrete analogue of Wiener measure with killing rate $m$.

$$
\begin{aligned}
& G_{m, g_{0}}^{c}\left(x_{1}, x_{2} ; y_{1}, y_{2}\right) \equiv \lim _{N, g_{0}^{\prime} \rightarrow 0}\left\langle\phi_{x_{1}}^{1} \phi_{x_{2}}^{1} ; \psi_{y_{1}}^{1} \psi_{y_{2}}^{1}\right\rangle \\
& =\sum_{\substack{\omega: x_{1} \rightarrow x_{2} \\
\omega^{\prime}: y_{1} \rightarrow y_{2}}} \iint d v_{\omega}^{m}(t) d v_{\omega^{\prime}}^{m}(s) I_{g_{0}}(t, s),
\end{aligned}
$$

where

$$
I_{g_{0}}(t, s)=\exp \left(-g_{0} \sum_{x} t_{x} s_{x}\right)-1
$$

Note that

$$
I_{g_{0}}(t, s)=0
$$

unless $\omega$ and $\omega^{\prime}$ intersect each other somewhere, in which case $-1<I_{g_{0}}(t, s)<0$, i.e. the functions $\exp \left(-g_{0} \sum_{x} t_{x} s_{x}\right)$ describe local, repulsive pair-interactions between random walks. By (1.9)-(1.11)

$$
g=g(m)=-\sum_{x} m^{d} \sum_{y, z} \chi^{-2} G_{m, g_{0}}^{c}(0, y ; x, z)
$$

with $\chi=\sum_{x} G_{m}(0, x)=m^{-2}$, is proportional to the intersection probability between simple random walks with killing rate $m$. The actual intersection probability is obtained from (1.12) by letting $g_{0}$ tend to $+\infty$ :

$$
\lim _{g_{0} \rightarrow+\infty}-\sum_{y, z} \chi^{-2} G_{m, g_{0}}^{c}(0, y ; x, z) \equiv P(0, x)
$$

where, by (1.7), (1.9), and (1.10),

$$
P(0, x)=\chi^{-2} \sum_{\substack{\omega: 0 \rightarrow-0 \\ \omega^{\prime}: x \rightarrow .}} \xi|\omega|+\left|\omega^{\prime}\right|+2 \chi\left(\omega \cap \omega^{\prime} \neq \phi\right)
$$

This is the intersection probability for two simple random walks, $\omega$ and $\omega^{\prime}$, starting at $0, x$, respectively. Since $P(0, x)$ is a pure number, and $m$ has the dimension of $[\text { length }]^{-1}$, the average intersection probability is given by

$$
P=\sum_{x} m^{d} P(0, x)=\left.g(m)\right|_{g_{0} \rightarrow \infty}
$$

If (1.5) were rigorously valid we would conclude that

and

$$
g(m) \sim|\log m|^{-1}, \text { as } m \searrow 0, \quad \text { for } d=4,
$$

$$
g(m) \underset{m \rightarrow 0}{\longrightarrow} g^{*}=\text { const } \varepsilon, \quad \text { for } d=4-\varepsilon .
$$


We note that, in the sense of dimensional analysis ${ }^{1}$ ), $d$ is a continuous parameter, and it is quite clear what is meant by $\mathbb{Z}^{4-\varepsilon}$. More rigorously, one may fix $d$, e.g. $d=3$, and study intersection probabilities for walks with jumping probabilities

$$
P_{x y}=-\left[\left(-\Delta+m^{2}\right)^{y}\right]_{x y}, \quad x \neq y,
$$

with $0<\gamma<1$. We note that $P_{x y}>0$, and $\left[\left(-\Delta+m^{2}\right)^{-\gamma}\right]_{x y}=\sum_{\omega: x \rightarrow y} P_{\omega} \xi^{|\omega|+1}$, where $\xi=\left[\left(-\Delta+m^{2}\right)^{\gamma}\right]_{x x}^{-1}, P_{\omega}=\prod_{(u, v) \in \omega} P_{u v}$. Then $d=4, \gamma=1$ is easily seen to be equivalent to $d=d_{0}, \gamma=d_{0} / 4$, e.g. $d=3, \gamma=3 / 4$, in the sense of dimensional analysis. Moreover $d=4-\varepsilon, \gamma=1$ corresponds to $d=3, \gamma=(3+\varepsilon) / 4$.

We now summarize how much of (1.15) can be proven rigorously, using a rigorous version of the flow equation (1.5):

A) In four dimensions,

$$
c_{1}|\ln m|^{-1} \leqq g(m) \leqq c_{2}|\ln m|^{-1}
$$

for small $m$. with

B) In $d=4-\varepsilon$ dimensions, there exists a non-trivial fixed point $g^{*}=\lim _{m \searrow 0} g(m)$,

$$
c_{3} \varepsilon \leqq g^{*} \leqq c_{4} \varepsilon
$$

for small $\varepsilon$.

Here $c_{1}, \ldots, c_{4}$ are finite, positive constants.

Similar results can be proven for the probability of intersection in one point of three random walks, with $d=4$ replaced by $d=3$; see also [1].

The lower bound in A) is due to Erdös and Taylor [3]. The upper bound in A) was first proven by Lawler [1]. In this paper we present a simple proof of it, motivated by the field-theoretic arguments leading to (1.5).

Our paper is organized as follows: In Sect. 2, we recall the proof of the lower bound on $g(m)$. In Sect. 3, we derive estimates on the $\beta$-function which imply the upper bounds on $g(m)$. Our estimates are based on the results of Sect. 2. In Sect. 4, we sketch some extensions of our results and draw conclusions.

\section{The Lower Bound}

The lower bound (1.17) on the average intersection probability was proven in four dimensions by Erdös and Taylor [3]. We present here a variant of their proof of this result, due to Sokal [12]. [For another proof see also Sect. 4, (a).]

Theorem 2.1. In four dimensions

$$
g \geqq c_{1}\left(\ln \frac{1}{m}\right)^{-1} .
$$

1 I.e. naive power counting 
In $d=4-\varepsilon$ dimensions

$$
g \geqq c_{3} \varepsilon .
$$

The proof of this theorem rests on the following ("skeleton"-)inequalities.

Lemma 2.2. There exist constants $d_{1}$ and $d_{2}$ such that, for small $m$,

$$
\begin{gathered}
P(0, x) \leqq d_{1} \sum_{z} G_{m}(0, z) G_{m}(z, x), \\
P(0, x) \geqq \frac{d_{2}}{B(m)}\left(2 \sum_{z} G_{m}(0, z) G_{m}(z, x)-\frac{1}{B(m)} \sum_{z_{1}, z_{2}} G_{m}\left(0, z_{1}\right) G_{m}\left(z_{1}, z_{2}\right)^{2} G_{m}\left(z_{2}, x\right)\right),
\end{gathered}
$$

where $B(m)=\sum_{z} G_{m}(0, z)^{2}$.

Proof of Lemma 2.2. In order to prove the upper bound, we note that

$$
\chi\left(\omega \cap \omega^{\prime} \neq \phi\right) \leqq \sum_{z} \chi\left(\omega \cap \omega^{\prime} \ni z\right)
$$

Splitting the walks $\omega$ and $\omega^{\prime}$ at the site $z$ and re-summing over all resulting walks yields

$$
P(0, x) \leqq \sum_{z} \xi^{-2} m^{4} \sum_{\substack{\omega_{1}: 0 \rightarrow z \\ \omega_{2}: z \rightarrow x \\ \omega_{3}: z \rightarrow-1 \\ \omega_{4}: z \rightarrow-}} \prod_{i=1}^{4} \xi^{\left|\omega_{i}\right|+1} \leqq \xi^{-2} \sum_{z} G_{m}(0, z) G_{m}(z, x),
$$

and we have used that

$$
\chi=m^{-2}=\sum_{z} G_{m}(0, z)=\sum_{\omega: 0 \rightarrow \cdot} \xi^{|\omega|+1} .
$$

In order to prove the lower bound, we choose some sublattice, $L$, of $\mathbb{Z}^{d}$. Then, by the inclusion-exclusion principle,

$$
\begin{aligned}
P(0, x) \geqq & \sum_{\substack{\omega: 0 \rightarrow . \\
\omega^{\prime}: x \rightarrow \cdot}} m^{4} \xi|\omega|+\left|\omega^{\prime}\right|+2 \chi\left(\omega \cap \omega^{\prime} \cap L \neq \phi\right) \\
\geqq & \sum_{z \in L} \sum_{\substack{\omega: 0 \rightarrow 0 \\
\omega^{\prime}: x \rightarrow \cdot}} m^{4} \xi|\omega|+\left|\omega^{\prime}\right|+2 \chi\left(\omega \cap \omega^{\prime} \cap L \ni z\right) \\
& -\frac{1}{2 !} \sum_{\substack{z_{1}, z_{2} \\
z_{1} \neq z_{2} \in L}} \sum_{\substack{\omega: 0 \rightarrow 0 \\
\omega^{\prime}: x \rightarrow 0}} m^{4} \xi^{|\omega|+\left|\omega^{\prime}\right|+2} \chi\left(\omega \cap \omega^{\prime} \cap L \ni z_{1}, z_{2}\right) .
\end{aligned}
$$

The probability that a walk $\omega$ starting at $x$ hits a site $y$ is given by

$$
g_{m}(x, y) \equiv \sum_{\omega: x \rightarrow \cdot} m^{2} \xi^{|\omega|+1} \chi(\omega \ni y)=G_{m}(x, y) / G_{m}(y, y)
$$


Thus

$P(0, x) \geqq \sum_{z \in L} g_{m}(0, z) g_{m}(z, x)-\sum_{\substack{z_{1}, z_{2} \in L \\ z_{1} \neq z_{2}}} g_{m}\left(0, z_{1}\right) g_{m}\left(z_{1}, z_{2}\right)^{2}\left(g_{m}\left(z_{1}, x\right)+g_{m}\left(z_{2}, x\right)\right)$.

It is convenient to choose $L$ to be a percolation lattice, i.e. we assign a probability $p$ that a site $x$ is in $L$ and a probability $(1-p)$ that $x \notin L$. Taking the expectation value on both sides of (2.9) we obtain

$$
\begin{aligned}
P(0, x) \geqq & \left(G_{m}(0,0)^{-2} p-G_{m}(0,0)^{-4} p^{2} B(m)\right) \sum_{z} G_{m}(0, z) G_{m}(z, x) \\
& -G_{m}(0,0)^{-4} p^{2} \sum_{z_{1}, z_{2}} G_{m}\left(0, z_{1}\right) G_{m}\left(z_{1}, z_{2}\right)^{2} G_{m}\left(z_{2}, x\right),
\end{aligned}
$$

which is equivalent to (2.4) if we choose

$$
p=\frac{G_{m}(0,0)^{2}}{3 B(m)}<1, \text { for } m \text { small enough. }
$$

Proof of Theorem 2.1. Inserting (2.10) in the definition (1.14) of $g(m)$ and using (2.7) we get

$$
g(m)>2 \frac{d^{2}}{B(m)} m^{d-4} \text {. }
$$

But since

$$
B(m) \sim \begin{cases}\ln \left(\frac{1}{m}\right), & d=4 \\ \frac{1}{\varepsilon} m^{-\varepsilon}, & d=4-\varepsilon,\end{cases}
$$

the theorem is proven. [See also Sect. 4, (a).]

\section{The Upper Bound}

We now turn to the main part of this paper: The proof of the upper bound on the intersection probability. Our proof is based on the following lemma, which relates the $\beta$-function to the average probability that two independent, simple random walks starting at the same point both intersect a third walk. We set $g=g(m)$.

Lemma 3.1. In $d=4-\varepsilon$ dimensions, $\varepsilon \geqq 0$,

$$
m \frac{d g}{d m}=-\varepsilon g+4 m^{d} \sum_{x} Q(0, x),
$$

where

$$
Q(0, x)=m^{6} \sum_{\substack{\omega_{1}: 0 \rightarrow . \\ \omega_{2}: 0 \rightarrow \\ \omega_{3}: x \rightarrow}} \prod_{i=1}^{3} \xi\left|\omega_{i}\right|+1 \chi\left(\omega_{1} \cap \omega_{3} \neq \phi\right) \chi\left(\omega_{2} \cap \omega_{3} \neq \phi\right) .
$$


Proof. Starting from the definition of $g$,

$$
g=m^{d+4} \sum_{\substack{\omega: 0 \rightarrow . \\ \omega^{\prime}: \rightarrow \rightarrow}} \xi^{|\omega|+\left|\omega^{\prime}\right|+2} \chi\left(\omega \cap \omega^{\prime} \neq \phi\right), \quad \xi=\frac{1}{2 d+m^{2}}
$$

we calculate the derivative, using $\frac{d \xi}{d m}=-2 \xi^{2} m$. Thus

$$
\begin{aligned}
m \frac{d g}{d m} & =(d+4) g-2 m^{d+6} \sum_{\substack{\omega: 0 \rightarrow-0 \\
\omega^{\prime}: \rightarrow \rightarrow \cdot}}\left(|\omega|+\left|\omega^{\prime}\right|+2\right) \xi^{|\omega|+\left|\omega^{\prime}\right|+3} \chi\left(\omega \cap \omega^{\prime} \neq \phi\right) \\
& =(d+4) g-4 m^{d+6} \sum_{\substack{\omega: 0 \rightarrow \cdot \\
\omega^{\prime}: \bullet \rightarrow \rightarrow .}}(|\omega|+1) \xi^{|\omega|+\left|\omega^{\prime}\right|+3} \chi\left(\omega \cap \omega^{\prime} \neq \phi\right),
\end{aligned}
$$

since we can interchange $\omega$ and $\omega^{\prime}$. Note that $|\omega|+1$ is the number of points that $\omega$ hits (counted with multiplicity). In each of these points we can split the walk $\omega$ into two independent walks $\omega_{1}$ and $\omega_{2}$. We thus have (with $\omega^{\prime}=\omega_{3}$ ):

$$
m \frac{d g}{d m}=(d+4) g-4 m^{d+6} \sum_{z} \sum_{\substack{\omega_{1}: 0 \rightarrow z \\ \omega_{2}: z \rightarrow z^{\prime} \\ \omega_{3}: \bullet^{\prime}}} \prod_{i=1}^{3} \xi^{\left|\omega_{i}\right|+1} \chi\left(\omega_{1} \cap \omega_{3} \neq \phi \text { or } \omega_{2} \cap \omega_{3} \neq \phi\right) \text {. }
$$

A moment of reflection shows that

$$
\begin{aligned}
\chi\left(\omega_{1} \cap \omega_{3} \neq \phi \text { or } \omega_{2} \cap \omega_{3} \neq \phi\right)= & \chi\left(\omega_{1} \cap \omega_{3} \neq \phi\right)+\chi\left(\omega_{2} \cap \omega_{3} \neq \phi\right) \\
& -\chi\left(\omega_{1} \cap \omega_{3} \neq \phi \text { and } \omega_{1} \cap \omega_{3} \neq \phi\right) .
\end{aligned}
$$

Inserting this identity on the right-hand side of (3.5) we have, after translation of $z$ to the origin,

$$
m \frac{d g}{d m}=(d+4) g-8 g+4 m^{d+6} \sum_{\substack{\omega_{1}: 0 \rightarrow \rightarrow \\ \omega_{2}: 0 \rightarrow \\ \omega_{3}: \rightarrow \rightarrow .}} \prod_{i=1}^{3} \xi\left|\omega_{i}\right|+1 \chi\left(\omega_{1} \cap \omega_{3} \neq \phi\right) \chi\left(\omega_{2} \cap \omega_{3} \neq \phi\right)
$$

where the term $-8 g$ on the right-hand side of (3.7) results from the first and second term on the right-hand side of (3.6), by re-summing over $\omega_{2}, \omega_{1}$, respectively, and adding the two contributions.

We now proceed to proving our main estimates.

Theorem 3.2 In four dimensions,

$$
g(m) \leqq c_{2}|\ln m|^{-1} .
$$

In $d=4-\varepsilon$ dimensions,

$$
g^{*} \leqq c_{4} \varepsilon,
$$

where $g^{*}=\lim _{m \searrow 0} g(m)$. 
Proof. Defining

$$
\beta(g, m) \equiv m \frac{d g}{d m}=-\frac{d g}{d|\ln m|}
$$

we need a lower bound on $\beta(\mathrm{g}, m)$, in order to obtain upper bounds on $g(m)$ and $g^{*}$, by integrating (3.10). By the Schwarz inequality,

$$
\begin{aligned}
& Q(0, x)=m^{2} \sum_{\omega_{3}: x \rightarrow .} \xi^{\left|\omega_{3}\right|+1}\left(m^{2} \sum_{\omega: 0 \rightarrow .} \xi|\omega|+1 \chi\left(\omega \cap \omega_{3} \neq \phi\right)\right)^{2} \\
& \geqq\left(m^{4} \sum_{\substack{\omega_{3}: x \rightarrow . \\
\omega: 0 \rightarrow .}} \xi\left|\omega_{3}\right|+|\omega|+2 \chi\left(\omega \cap \omega_{3} \neq \phi\right)\right)^{2}=P(0, x)^{2},
\end{aligned}
$$

and we have used that

$$
m^{2}=\chi^{-1}=\left(\sum_{\omega: 0 \rightarrow} \xi^{|\omega|+1}\right)^{-1}
$$

We define $B_{R}=\left\{x \in \mathbb{Z}^{d}|| x \mid \leqq R\right\}$, and use the Schwarz inequality once more. This yields

$$
\beta(g, m) \geqq-\varepsilon g+4 m^{d} \sum_{x \in B_{R}} P(0, x)^{2} \geqq-\varepsilon g+\frac{4 m^{-d}}{\left|B_{R}\right|}\left(m^{d} \sum_{x \in B_{R}} P(0, x)\right)^{2},
$$

where $\left|B_{R}\right|$ denotes the number of sites in $B_{R}$. In order to compare $m^{d} \sum_{x \in B_{R}} P(0, x)$ to $g=m^{d} \sum_{x} P(0, x)$, we need an upper bound on $P(0, x)$, for $|x|>R$ : For $x=\left(x_{1}, \ldots, x_{d}\right) \in \mathbb{Z}^{d}$, let $x_{\alpha}$ be the coordinate of $x$ with the largest absolute value. Thus $\left|x_{\alpha}\right| \geqq d^{-1 / 2}|x|$. Let $\pi_{x}$ be the lattice plane perpendicular to the $\alpha$-axis and intersecting that axis in the point $\left[x_{\alpha} / 2\right] e_{\alpha}$, where $[a]$ is the largest integer $\leqq a$, and $e_{\alpha}$ is the unit lattice vector in the $\alpha$-direction. We now recall that $P(0, x)$ is the probability that a random walk $\omega$ starting at 0 and a random walk $\omega^{\prime}$ starting at $x$ intersect in a common point of $\mathbb{Z}^{d}$. This probability is clearly bounded above by the probability, $R(0, x)$, that either $\omega$ hits the plane $\pi_{x}$, or $\omega^{\prime}$ hits $\pi_{x}$. (For if neither $\omega$, nor $\omega^{\prime}$ hit $\pi_{x}$, then $\omega$ and $\omega^{\prime}$ will always be on different sides of $\pi_{x}$ and hence never intersect.) Thus

$$
P(0, x) \leqq R(0, x) \leqq c T(0, x)
$$

where

$$
T(0, x)=\operatorname{Prob}\left\{\omega \mid \omega: 0 \rightarrow \cdot, \omega \text { hits } \pi_{x}\right\}
$$

(and $c=2$ if $x_{\alpha}$ is even). Now, $T(0, x)$ can easily be calculated explicitly:

$$
T(0, x)=\sum_{z \in \pi_{x}}\left[G_{m}\left(0, z-\left(\operatorname{sign} x_{\alpha}\right) e_{\alpha}\right)-G_{m}\left(0, z+\left(\operatorname{sign} x_{\alpha}\right) e_{\alpha}\right)\right]
$$


By using the Fourier representation of $G_{m}$ and the calculus of residues to carry out the momentum space integration we find

$$
0<T(0, x) \leqq c^{\prime} e^{-c^{\prime \prime} m\left|x_{\alpha}\right|} \leqq c^{\prime} \exp \left(-c^{\prime \prime} d^{-1 / 2} m|x|\right) .
$$

Thus $P(0, x) \leqq \bar{c} \exp (-\tilde{c} m|x|)$, from which we get

$$
m^{d} \sum_{|x|>R} P(0, x) \leqq C e^{-\tilde{c} m R},
$$

for some finite constants $C$ and $\tilde{c}$.

We now discuss separately the cases $d=4$ and $d=4-\varepsilon$.

(i) For $d=4$, we choose

$$
R=(\tilde{c} m)^{-1} \ln \left(\frac{2 C}{c_{1}}|\ln m|\right)
$$

where $c_{1}$ is the constant appearing in Theorem 2.1, and $\tilde{c}, C$ are the constants of estimate (3.16). Then, by (2.1) and (3.16),

$$
m^{4} \sum_{|x|>R} P(0, x) \leqq c_{1}(2|\ln m|)^{-1} \leqq \frac{1}{2} g,
$$

and thus

$$
m^{4} \sum_{x \in B_{R}} P(0, x) \geqq \frac{1}{2} g .
$$

With (3.11) and (3.12) this yields

$$
m \frac{d g}{d m}=\beta(g, m) \geqq \text { const } g^{2}(\ln |\ln m|)^{-d},
$$

and after integration,

$$
g(m)^{-1}-g\left(m_{0}\right)^{-1} \geqq \mathrm{const} \int_{\left|\ln m_{0}\right|}^{|\ln m|} d \tau(\ln \tau)^{-d} .
$$

Since the right-hand side of (3.21) diverges, as $m \searrow 0$, we conclude that

$$
0 \leqq g(m) \leqq c_{2}|\ln m|^{-1}(\ln |\ln m|)^{4},
$$

in particular $g(m) \rightarrow 0$, as $m \rightarrow 0$.

With more effort, one can improve (3.22) and show that $g(m) \leqq c_{2}|\ln m|^{-1}$.

(ii) For $d=4-\varepsilon$, we choose in (3.16)

$$
R=(\tilde{c} m)^{-1} \ln \left(\frac{2 C}{c_{3} \varepsilon}\right)
$$

Then

$$
m^{d} \sum_{|x|>R} P(0, x) \leqq \frac{c_{3}}{2} \varepsilon \leqq \frac{1}{2} g
$$


by Theorem 2.1 , and hence

$$
m \frac{d g}{d m} \geqq \text { const } g^{2}|\ln \varepsilon|^{-d}-\varepsilon g,
$$

or

$$
\frac{d g}{d|\ln m|} \leqq \varepsilon g-\text { const } g^{2}|\ln \varepsilon|^{-d}
$$

Hence

$$
\lim _{m \searrow 0} g(m)=g^{*} \leqq c_{4} \varepsilon|\ln \varepsilon|^{d} .
$$

Note added in typescript. We now briefly outline how to remove the factors $(\ln |\ln m|)^{4},|\ln \varepsilon|^{d}$, respectively, by sharpening the bounds (3.12) and (3.16). Our argument is somewhat inspired by one used by M. Aizenman in his independent work on closely related problems. (We received his preprint after completion of the first draft of this paper.)

The simple estimate

$Q(0, x) \geqq m^{2} \sum_{\omega_{1}: 0 \rightarrow .} \xi^{\left|\omega_{1}\right|+1}\left(m^{2} \sum_{\omega_{2}: x \rightarrow .} \xi^{\left|\omega_{2}\right|+1} \chi\left(\omega_{1} \cap \omega_{2} \neq \phi\right)\right)^{2} \chi\left(\omega_{1} \cap \pi_{x} \neq \phi\right)$,

where $\pi_{x}$ is the plane defined after (3.12), implies, by the Schwarz inequality,

$$
\begin{aligned}
& \beta(g, m) \geqq-\varepsilon g+\left(m^{d} \sum_{x} m^{2} \sum_{\omega_{1}: 0 \rightarrow .} \xi^{\left|\omega_{1}\right|+1} \chi\left(\omega_{1} \cap \pi_{x} \neq \phi\right)\right)^{-1} \\
& \cdot\left\{m^{d} \sum_{x} m^{4} \sum_{\substack{\omega_{1}: 0 \rightarrow . \\
\omega_{2}: x \rightarrow .}} \xi\left|\omega_{1}\right|+\left|\omega_{2}\right|+2 \chi\left(\omega_{1} \cap \omega_{2} \neq \phi\right) \frac{1}{2}\left[\chi\left(\omega_{1} \cap \pi_{x} \neq \phi\right)+\chi\left(\omega_{2} \cap \pi_{x} \neq \phi\right)\right]\right\}^{2} \text {, }
\end{aligned}
$$

where use was made of the symmetry under interchange of $\omega_{1}$ and $\omega_{2}$. The denominator is just $m^{d} \sum_{x} T(0, x) \leqq$ const, by (3.15), and since

$$
\chi\left(\omega_{1} \cap \omega_{2} \neq \phi\right) \frac{1}{2}\left[\chi\left(\omega_{1} \cap \pi_{x} \neq \phi\right)+\chi\left(\omega_{2} \cap \pi_{x} \neq \phi\right)\right] \geqq \frac{1}{2} \chi\left(\omega_{1} \cap \omega_{2} \neq \phi\right),
$$

we get the lower bound

$$
\beta(g, m) \geqq-\varepsilon g+C_{d} g^{2}
$$

( $C_{d}$ is a positive constant), which, upon integration, yields the claim of the theorem.

\section{Extensions and Conclusions}

The following results can be established by using the techniques developed in our paper:

(a) In (1.9) we have defined a connected four-point function for the $g_{0}|\phi|^{2}|\psi|^{2}$-theory, for finite values of $g_{0}$, in the limit $N \rightarrow 0$. We have noted that, for finite $g_{0}$,

$$
P_{g_{0}}(0, x) \equiv-m^{4} \sum_{y, z} G_{m, g_{0}}^{c}(0, y ; x, z)
$$


is proportional, but strictly smaller, than the intersection probability, $P(0, x)$. Since the function $-I_{g_{0}}(t, s)=1-\exp \left(-g_{0} \sum_{x} t_{x} s_{x}\right)$ is monotone increasing in $g_{0}$, we conclude, using (1.9)-(1.11), that

$$
\begin{aligned}
& P_{g_{0}}(0, x) \text { is monotone increasing in } g_{0}, \text { with } \\
& \lim _{g_{0} \searrow 0} P_{g_{0}}(0, x)=0, \lim _{g_{0} \succ \infty} P_{g_{0}}(0, x)=P(0, x) .
\end{aligned}
$$

Clearly

$$
-I_{g_{0}}(t, s) \geqq-I_{g_{0}^{\prime}}(t, s) \geqq g_{0}^{\prime} \sum_{z} t_{z} s_{z}-\frac{g_{0}^{\prime 2}}{2} \sum_{z_{1}, z_{2}} t_{z_{1}} t_{z_{2}} S_{z_{1}} s_{z_{2}},
$$

For arbitrary $g_{0}^{\prime} \leqq g_{0}$. By the splitting lemma of [13], this yields

$$
\begin{aligned}
P_{g_{0}}(0, x) \geqq & g_{0}^{\prime} \sum_{z} G_{m}(0, z) G_{m}(x, z) \\
& -\frac{g_{0}^{\prime 2}}{2} \sum_{z_{1}, z_{2}} G_{m}\left(0, z_{1}\right) G_{m}\left(z_{1}, z_{2}\right)^{2} G_{m}\left(z_{1}, x\right) \\
& -\frac{g_{0}^{\prime 2}}{2} \sum_{z_{1}, z_{2}} G_{m}\left(0, z_{1}\right) G_{m}\left(z_{1}, z_{2}\right)^{2} G_{m}\left(z_{2}, x\right) .
\end{aligned}
$$

By choosing $g_{0}^{\prime}=B(m)^{-1} g_{0}$, we obtain

$$
\begin{aligned}
P_{g_{0}}(0, x) \geqq & \frac{g_{0}\left(1-g_{0} / 2\right)}{B(m)} \sum_{z} G_{m}(0, z) G_{m}(x, z) \\
& -\frac{c g_{0}}{B(m)^{2}} \sum_{z_{1}, z_{2}} G_{m}\left(0, z_{1}\right) G\left(z_{1}, z_{2}\right)^{2} G_{m}\left(z_{2}, x\right),
\end{aligned}
$$

with $B(m)$ as in Lemma 2.2 .

For $g_{0}$ small enough, this lower bound has the desired feature. But, since $P_{g_{0}}(0, x)$ is monotone increasing in $g_{0}$ we have an adequate lower bound for all values of $g_{0}$. Thus, Theorem 2.1 holds in this more general situation, too. It may be checked without difficulty that the results in Sect. 3 extend to the full range of values of $g_{0}$, as well.

Let $P_{\phi}(m)$ be the probability that two simple random walks starting at the origin never intersect after the first step. Lawler [1] has studied this quantity which provides a useful notion for the study of triviality of $g \phi_{4}^{4}$ theory. He has proven that

and

$$
P_{\phi}(m) \geqq\left\{\begin{array}{ll}
c_{\varepsilon} m^{\varepsilon}, & d=4-\varepsilon \\
c|\ln \mathrm{m}|^{-1}, & d=4
\end{array},\right.
$$

$$
P_{\phi}(m) \leqq \begin{cases}c_{\varepsilon}^{\prime} m^{\varepsilon / 2}, & d=4-\varepsilon \\ c^{\prime}|\ln m|^{-1 / 2}, & d=4\end{cases}
$$

These results can be recovered, in our formalism, from the following straightforward estimates.

$$
P_{\phi}(m)=1-\chi^{-2} P(0,0) \geqq 1-B(m) P_{\phi}(m),
$$


providing the lower bound, and

$$
P_{\phi}(m)^{2} \leqq \text { const } m^{4-d} g(m),
$$

which, with the estimates on $g(m)$, yields the upper bound.

In the context of the Edwards model closely related bounds were proven in [14]. It would be interesting to calculate the exact behaviour of $P_{\phi}(m)$, as $m \searrow 0$. A heuristic argument (based on an approximate flow equation for $P_{\phi}(m)$ ) suggests that, in $d=4-\varepsilon, P_{\phi}(m) \sim m^{\alpha}$, with $\frac{\varepsilon}{2}<\alpha<\varepsilon$. We thank A. Sokal and T. Spencer for very instructive discussions on the results reported in this paragraph.

(b) The results of Sects. 1-3 can be generalized to include estimates of the probability that $n$ simple random walks $\omega_{1}, \ldots, \omega_{n}$ intersect in a common point of $\mathbb{Z}^{d}, n=3,4, \ldots$. This problem, too, may be posed as a problem in Euclidean field theory by considering a lattice theory with action

$$
\begin{aligned}
& \mathscr{A}\left(\boldsymbol{\phi}_{1}, \ldots, \boldsymbol{\phi}_{n}\right) \\
& =\sum_{x \in \mathbb{Z}^{d}}\left\{\sum_{j} \frac{1}{2}\left(\left|\left(\nabla \boldsymbol{\phi}_{j}\right)_{x}\right|^{2}+m^{2}\left|\boldsymbol{\phi}_{j, x}\right|^{2}\right)+2^{-n} g_{0}\left|\boldsymbol{\phi}_{1, x}\right|^{2}\left|\boldsymbol{\phi}_{2, x}\right|^{2} \ldots\left|\boldsymbol{\phi}_{n, x}\right|^{2}\right\} .
\end{aligned}
$$

By the methods of $[6,10]$, the limits

$$
G_{m, g_{0}}^{c}\left(x_{1}, x_{1}^{\prime} ; \ldots ; x_{n}, x_{n}^{\prime}\right)=\lim _{g_{0} \rightarrow \infty} \lim _{N \rightarrow 0}\left\langle\phi_{1, x_{1}}^{1} \phi_{1, x_{1}^{\prime}}^{1} ; \ldots ; \phi_{n, x_{n}}^{1} \phi_{n, x_{n}^{\prime}}^{1}\right\rangle
$$

can be shown to exist, and the average intersection probability $g_{n}(m)$ for $n$ walks is given by

$$
g_{n}(m)=-\sum_{x_{2}, \ldots, x_{n}}\left(m^{d}\right)^{n-1} \sum_{x_{1}^{\prime}, \ldots, x_{n}^{\prime}} \chi^{-n} G_{m, g_{0}}^{c}\left(x_{1}, x_{1}^{\prime} ; \ldots ; x_{n}, x_{n}^{\prime}\right) .
$$

From these formulas it follows that the upper critical dimension is $d_{u}=2 n /(n-1)$, in particular, for $n=3, d_{u}=3$. For $d=d_{u}$, we have

$$
g_{n}(m) \sim|\log m|^{-1}, \quad \text { as } \quad m \searrow 0,
$$

as was first proven by Lawler [1]. Moreover, for a fixed value of $n$, one can estimate the behaviour in dimension $d_{u}-\varepsilon$, with $\varepsilon$ small. Our results suggest that there may be no non-trivial $g_{0} \phi^{6}$-theories in three dimensions, and that the violations of meanfield theory in tricritical point theory in three dimensions are logarithmic.

(c) Techniques related to the ones we have developed in this paper do also extend to theories like the $-g_{0} \operatorname{tr}\left(\phi^{3}\right)$ model, where $\phi$ is a hermitian $N \times N$ matrixfield, in the limit $N \rightarrow \infty$. For this model, one can establish, for example, asymptotic freedom $(\beta<0)$, for $d \leqq 6$. However, the consequences of our results are not terribly spectacular. A more interesting application concerns intersection probabilities of branched polymers without self-interactions: We can prove that two branched polymers without self-interactions do not meet each other, in the limit where the correlation length diverges, with probability 1 in $d \geqq 8$, and we have fairly sharp estimates for the intersection probability in dimension $d=8-\varepsilon$. Precise statements and proofs will appear elsewhere. 


\section{Conclusions}

We believe that the results and techniques reported in this paper have, at the very least, some pedagogical value: They show that renormalization group flow equations can, sometimes, make rigorous mathematical sense and yield precise results. However, the idea to use rigorous renormalization flow inequalities to analyze the critical behaviour of lattice models may turn out to actually have a much wider range of applications than the ones considered in this paper. In fact, this is suggested by results (c), above.

We have been informed that Michael Aizenman has also obtained results on intersection probabilities of random walks, using a $\beta$-function approach apparently related to ours.

Acknowledgements. We are indebted to D. Brydges, K. Gawedzki, T. Spencer, and K. Symanzik for very helpful discussions about different topics related to this paper. We are especially indebted to A. Sokal for sharing many insights with us and for permission to describe an unpublished proof of his.

This paper is dedicated to the memory of K. Symanzik, on whose deep ideas it is based.

\section{References}

1. Lawler, G.F.: The probability of intersection of independent random walks in four dimensions. Commun. Math. Phys. 86, 539 (1982); and Preprint, Duke University 1983

2. Dvoretzky, A., Erdös, P., Kakutani, S.: Acta Sci. Math. Szeged. 12, 75 (1950)

3. Erdös, P., Taylor, S.J.: Acta Math. Acad. Sci. Hung. 11, 137 and 231 (1960)

4. Glimm, J., Jaffe, A.: Quantum physics. Berlin, Heidelberg, New York: Springer 1981

5. Aizenman, M.: Geometric analysis of $\phi^{4}$ fields and Ising models. Parts I and II. Commun. Math. Phys. 86, 1 (1982)

Fröhlich, J.: On the triviality of $\lambda \varphi_{d}^{4}$ theories and the approach to the critical point in $d \geqq 4$ dimensions. Nucl. Phys. B200 [FS4], 281 (1982)

6. Symanzik, K.: Euclidean quantum field theory. In: Local quantum theory. Jost, R. (ed.). New York, London: Academic Press 1969

7. Fröhlich, J.: Quantum field theory in terms of random walks and random surfaces, Cargèse lecture notes 1983 (to be published). Aizenman, M.: Rigorous results on the critical behaviour in statistical mechanics. In: Scaling and Self-Similarity in Physics. Fröhlich, J. (ed.). Boston, Basel, Stuttgart: Birkhäuser 1983

Brydges, D.: Field theories and Symanzik's polymer representation. In: Gauge theories: fundamental interactions and rigorous results. Dita, P. et al. (eds.). Boston, Basel, Stuttgart: Birkhäuser 1983

8. Amit, D.: Field theory, renormalization group and critical phenomena. New York: McGrawHill 1978

9. Brézin, E., Le Guillou, J., Zinn-Justin. J.: Discussion of critical phenomena for general $n$-vector models. Phys. Rev. B10, 892 (1974)

10. Brydges, D., Fröhlich, J., Spencer, T.: The random walk representation of classical spin systems and correlation inequalities. Commun. Math. Phys. 83, 123 (1982)

11. Brydges, D., Fröhlich, J., Sokal, A.: Unpublished

12. Sokal. A.: Private communication

13. Brydges, D., Fröhlich, J., Sokal, A.: The random walk representation of classical spin systems and correlation inequalities. II. The skeleton inequalities. Commun. Math. Phys. 91, 117 (1983)

14. Bovier, A., Felder, G., Fröhlich, J.: On the critical properties of the Edwards and the selfavoiding walk model of polymer chains. Nucl. Phys. B230 [FS 10], 119 (1984)

Communicated by A. Jaffe

Received March 19, 1984; in revised form April 30, 1984 